\title{
Article \\ On the Estrada Indices of Unicyclic Graphs with Fixed Diameters
}

\author{
Wenjie Ning ${ }^{1}\left(\mathbb{D}\right.$ and Kun Wang ${ }^{2, *}$ \\ 1 College of Science, China University of Petroleum (East China), Qingdao 266580, China; 20180007@upc.edu.cn \\ 2 College of Mathematics and Systems Science, Shandong University of Science and Technology, \\ Qingdao 266590, China \\ * Correspondence: skd996195@sdust.edu.cn
}

check for

updates

Citation: Ning, W.; Wang, K. On the Estrada Indices of Unicyclic Graphs with Fixed Diameters. Mathematics 2021, 9, 2395. https://doi.org/ $10.3390 /$ math 9192395

Academic Editor: David Dolžan

Received: 4 September 2021

Accepted: 20 September 2021

Published: 26 September 2021

Publisher's Note: MDPI stays neutral with regard to jurisdictional claims in published maps and institutional affiliations.

Copyright: (c) 2021 by the authors. Licensee MDPI, Basel, Switzerland. This article is an open access article distributed under the terms and conditions of the Creative Commons Attribution (CC BY) license (https:// creativecommons.org/licenses/by/ $4.0 /)$.

\begin{abstract}
The Estrada index of a graph $G$ is defined as $E E(G)=\sum_{i=1}^{n} e^{\lambda_{i}}$, where $\lambda_{1}, \lambda_{2}, \ldots, \lambda_{n}$ are the eigenvalues of the adjacency matrix of $G$. A unicyclic graph is a connected graph with a unique cycle. Let $\mathcal{U}(n, d)$ be the set of all unicyclic graphs with $n$ vertices and diameter $d$. In this paper, we give some transformations which can be used to compare the Estrada indices of two graphs. Using these transformations, we determine the graphs with the maximum Estrada indices among $\mathcal{U}(n, d)$. We characterize two candidate graphs with the maximum Estrada index if $d$ is odd and three candidate graphs with the maximum Estrada index if $d$ is even.
\end{abstract}

Keywords: adjacency matrix; Estrada index; unicyclic graph; spectral moment; diameter

\section{Introduction}

In this paper, we only consider simple undirected graphs. Let $G=(V(G), E(G))$ be a graph with $n$ vertices and $m$ edges. Let $N_{G}(v)$ be the set of vertices adjacent to $v$ in $G$. The degree of $v$ in $G$, denoted by $d_{G}(v)$, is equal to $\left|N_{G}(v)\right|$. A vertex of degree one is called a pendant vertex. The edge incident with a pendant vertex is known as a pendant edge. Let $S \neq \varnothing \subseteq V(G)$. Then denote by $G[S]$ the subgraph induced by $S$. If $D \subseteq E(G)$ (or $D \subseteq V(G)$ ), then we write $G-D$ for the graph obtained from $G$ by deleting all of its edges (or vertices, resp.) in $D$. If $D \subseteq E(\bar{G})$, then we denote by $G+D$ the graph obtained from $G$ by adding all of edges in $D$ to the graph.

Let $A(G)$ be the adjacency matrix of $G$. Denote the eigenvalues of $A(G)$ by $\lambda_{1}, \lambda_{2}, \ldots, \lambda_{n}$ and assume $\lambda_{1} \geq \lambda_{2} \geq \ldots \geq \lambda_{n}$. Then $\lambda_{1}$, usually denoted by $\rho(G)$, is called the spectral radius of $G$. The Estrada index of $G$ is defined as

$$
E E(G)=\sum_{i=1}^{n} e^{\lambda_{i}}
$$

This graph invariant was first proposed as a measure of the degree of folding of a protein [1] and now has been found multiple applications in various fields, such as measurements of the subgraph centrality and the centrality of complex networks $[2,3]$ and the extended molecular branching [4]. Recently, the correlation between the Estrada index and $\pi$-electronic energies for benzenoid hydrocarbons was investigated in [5], the results of which warrant its further usage in quantitative structure-activity relationships. Given these prominent applications of the Estrada index, the research on it is of theoretical and practical significance. In the last few decades, some mathematical properties of the Estrada index, including various bounds for it, have been established [6-12].

In 1986, Brualdi and Solheid [13] proposed the following problem concerning the spectral radii of graphs: Given a set $\mathcal{G}$ of graphs, find an upper bound for the spectral radius of graphs in $\mathcal{G}$ and characterize the graphs for which the maximal spectral radius is attained. The corresponding problem of a given graph invariant has been widely studied 
(see [14-16], for example). Motivated by this, many results have been obtained on characterizing graphs that maximize (or minimize) the Estrada index among a given set of graphs. For example, some interesting results were obtained for the general trees [17], trees with a given matching number [18], trees with a fixed diameter [19], trees with perfect matching and a fixed maximum degree [20], and trees with a fixed number of pendant vertices [21]. Du and Zhou [22] showed a graph with the maximal Estrada index and two candidate graphs with the minimum Estrada index among all unicyclic graphs. Moreover, they determined the unique graphs with maximum Estrada indices among graphs with given parameters [23]. Wang et al. [24] and Zhu et al. [25] characterized the bicyclic graph and the tricyclic graph with maximum Estrada indices, respectively. E. Andrade et al. [26] presented the graph having the largest Estrada index of its line graph among all graphs on $n$ vertices with connectivity less than or equal to a fixed number. For more results on the Estrada index and its variations, the readers may refer to [27-30].

A unicyclic graph is a connected graph with a unique cycle. Let $P_{n}$ and $C_{n}$ be the path and the cycle on $n$ vertices, respectively. Denote by $\mathcal{U}(n, d)$ the set of all unicyclic graphs with $n$ vertices and diameter $d$. In this paper, we characterize the graphs with the maximum Estrada index in $\mathcal{U}(n, d)$.

This paper is organized as follows. In Section 2, we list some transformations which can be used to compare the Estrada indices of two graphs. In Section 3, we determine the graphs with the maximum Estrada index among unicyclic graphs in $\mathcal{U}(n, d)$. We show two candidate graphs with the maximal Estrada index if $d$ is odd and three candidate graphs with the maximal Estrada index if $d$ is even. We also propose a hypothesis on the structure of the extremal graph with the maximum Estrada index in $\mathcal{U}(n, d)$.

\section{Preliminaries}

In order to obtain the main results of this paper, we give some definitions and lemmas here.

A walk of length $k$ in a graph $G$ is any sequence of vertices and edges in $G$, $W=v_{1} e_{1} v_{2} e_{2} \cdots v_{k} e_{k} v_{k+1}$, such that $e_{i}=v_{i} v_{i+1}$ for every $1 \leq i \leq k$. For a subsequence $v_{i} e_{i} v_{i+1} \cdots v_{j-1} e_{j-1} v_{j}$ of $W$, we refer to it as a $\left(v_{i}, v_{j}\right)$-section of $W$. Usually, we write $W=v_{1} v_{2} \cdots v_{k+1}$ instead for simplicity and call it a $\left(v_{1}, v_{k+1}\right)$-walk. Let $\vec{W}=v_{k+1} v_{k} \cdots v_{1}$. Then $\vec{W}$ is called the reverse of $W$. If $v_{1}=v_{k+1}$, then $W$ is called a closed walk.

Let $M_{k}(G)$ be the $k$ th spectral moment of the graph $G$ defined as $M_{k}(G)=\sum_{i=1}^{n} \lambda_{i}^{k}$. It is well-known that $M_{k}(G)$ equals the number of closed walks of length $k$ in $G$; see [31]. Then by the Taylor expansion of the exponential function $e^{x}$, we have

$$
E E(G)=\sum_{k=0}^{\infty} \frac{M_{k}(G)}{k !}
$$

Let $G$ and $H$ be two graphs with $x, y \in V(G), u, v \in V(H)$ and $e \in E(G)$. Suppose $k$ is an arbitrary positive integer. Let $W_{k}(G ; x,[e])$ be the set of all $(x, x)$-walks of length $k$ going through the edge $e$ in $G$ and let $\left|W_{k}(G ; x,[e])\right|=M_{k}(G ; x,[e])$. Let $W_{k}(G ; x, y)$ be the set of all $(x, y)$-walks of length $k$ in $G$ and let $\left|W_{k}(G ; x, y)\right|=M_{k}(G ; x, y)$. If $M_{k}(G ; x, y) \leq$ $M_{k}(H ; u, v)$ for all positive integers $k$, then we write $(G ; x, y) \preceq(H ; u, v)$. If $(G ; x, y) \preceq$ $(H ; u, v)$, and $M_{k_{0}}(G ; x, y)<M_{k_{0}}(H ; u, v)$ for some positive integer $k_{0}$, then we write $(G ; x, y) \prec(H ; u, v)$. For convenience, let $W_{k}(G ; x)=W_{k}(G ; x, x), M_{k}(G ; x)=M_{k}(G ; x, x)$ and $(G ; u)=(G ; u, u)$.

The following four results are often used to compare the Estrada indices of two graphs.

Lemma 1 ([28]). Let $H$ be a graph (not necessarily connected) with $u, v \in V(H)$. Suppose that $w_{i} \in V(H)$, and $u w_{i}, v w_{i} \notin E(H)$ for $1 \leq i \leq r$. Let $E_{u}=\left\{u w_{1}, u w_{2}, \ldots, u w_{r}\right\}$ and $E_{v}=\left\{v w_{1}, v w_{2}, \ldots, v w_{r}\right\}$. Let $H_{u}=H+E_{u}$ and $H_{v}=H+E_{v}$. If $(H ; u) \prec(H ; v)$ and $\left(H ; w_{i}, u\right) \preceq\left(H ; w_{i}, v\right)$ for $1 \leq i \leq r$, then $E E\left(H_{u}\right)<E E\left(H_{v}\right)$. 
Lemma 2 ([32]). Let $H_{1}$ and $H_{2}$ be two non-trivial graphs with $u, v \in V\left(H_{1}\right), w \in V\left(H_{2}\right)$. Let $G_{u}$ be the graph obtained from $H_{1}$ and $H_{2}$ by identifying $u$ with $w$, and $G_{v}$ be the graph obtained from $H_{1}$ and $H_{2}$ by identifying $v$ with w. If $\left(H_{1} ; v\right) \prec\left(H_{1} ; u\right)$, then $E E\left(G_{v}\right)<E E\left(G_{u}\right)$.

Lemma 3 ([32]). Let $G_{1}$ and $G_{2}$ be two connected graphs with $u \in V\left(G_{1}\right)$ and $v \in V\left(G_{2}\right)$. Let $G$ be the graph obtained by joining $u$ and $v$ with and edge, and let $G^{\prime}$ be the graph obtained by identifying $u$ with $v$, and attaching a pendant vertex to the common vertex. If $d_{G}(u), d_{G}(v) \geq 2$, then $E E(G)<E E\left(G^{\prime}\right)$.

Theorem 1 ([27]). Let $G$ be a connected graph and $G_{u, v}(p, q)$ be the graph obtained from $G$ by attaching $p$ and $q$ pendant edges to $u$ and $v$, respectively, where $u, v \in V(G)$ and $p, q \geq 1$. Then $E E\left(G_{u, v}(p+q, 0)\right) \geq E E\left(G_{u, v}(p, q)\right)$ or $E E\left(G_{u, v}(0, p+q)\right) \geq E E\left(G_{u, v}(p, q)\right)$. Furthermore, $E E\left(G_{u, v}(p+q, 0)\right)>E E\left(G_{u, v}(p, q)\right)$ if $d_{G}(u) \geq d_{G}(v)$ or $E E\left(G_{u, v}(0, p+q)\right)>$ $E E\left(G_{u, v}(p, q)\right)$ if $d_{G}(v) \geq d_{G}(u)$.

\section{Lemmas}

In this section, we give some lemmas that can be used to prove $(G ; v) \prec(G ; u)$ in a graph $G$, where $u, v \in V(G)$.

Lemma 4. Let $G$ be a simple graph and $u, v \in V(G)$. If $N_{G}(v) \subseteq N_{G}(u)$, then $(G ; v) \preceq(G ; u)$, and $(G ; w, v) \preceq(G ; w, u)$ for each $w \in V(G)$. Moreover, if $d_{G}(v)<d_{G}(u)$, then $(G ; v) \prec(G ; u)$.

Proof of Lemma 4. Since $G$ is simple, $N_{G}(v) \subseteq N_{G}(u)$ implies $u v \notin E(G)$. Let $k \geq 0$ and $W \in W_{k}(G ; v)$. Then $W$ can be written as $W=v w_{1} \cdots w_{2} v$, where $w_{1}, w_{2} \in N_{G}(v)$. Let $\widehat{W}=u w_{1} \cdots w_{2} u$. Since $N_{G}(v) \subseteq N_{G}(u)$ and $u v \notin E(G)$, the map $f_{k}: W_{k}(G ; v) \rightarrow W_{k}(G ; u)$, defined as $f_{k}(W)=\widehat{W}$ is an injection. Thus, $M_{k}(G ; v) \leq M_{k}(G ; u)$. Since $k \geq 0$ is arbitrary, we get $(G ; v) \preceq(G ; u)$. Note that $d_{G}(v)=M_{2}(G ; v)$ and $d_{G}(u)=M_{2}(G ; u)$. Therefore, $(G ; v) \prec(G ; u)$ if $d_{G}(v)<d_{G}(u)$ further holds. Similarly, we can show $(G ; w, v) \preceq(G ; w, u)$ for each $w \in V(G)$.

Lemma 5. Let $G$ be a graph and $H=G+$ e such that $e=u v \in E(\bar{G})$. If $(G ; v) \preceq(G ; u)$, then $(H ; v) \preceq(H ; u)$. Moreover, if $(G ; v) \prec(G ; u)$, then $(H ; v) \prec(H ; u)$.

Proof of Lemma 5. For each $z \in\{u, v\}$ and $k \geq 0$, by the definition of $M_{k}(H ; z)$,

$$
M_{k}(H ; z)=M_{k}(G ; z)+M_{k}(H ; z,[e])
$$

Since $(G ; v) \preceq(G ; u)$, we have $M_{k}(G ; v) \leq M_{k}(G ; u)$. Therefore, there exits an injection $f_{k}: W_{k}(G ; v) \rightarrow W_{k}(G ; u)$. In order to prove $M_{k}(H ; v) \leq M_{k}(H ; u)$, it suffices to show $M_{k}(H ; v,[e]) \leq M_{k}(H ; u,[e])$.

Let $W \in W_{k}(H ; v,[e])$. Then either veu or uev must be contained in $W$. If $W$ does not contain the section $u e v$, or veu appears earlier than uev in $W$, then $W$ can be decomposed uniquely to $W=W_{1} e W_{2}$ such that $W_{1} \in W_{k_{1}}(G ; v)$ for some $k_{1} \geq 0$ and $W_{2} \in W_{k_{2}}(H ; u, v)$ for some $k_{2} \geq 0$. In this case, we define $h_{k}(W)=f_{k_{1}}\left(W_{1}\right) e \overleftarrow{W_{2}}$. Then $h_{k}(W) \in W_{k}(H ; u,[e])$.

If $W$ does not contain the section veu, or uev appears earlier than veu in $W$, then $W$ can be decomposed uniquely to $W=W_{1} e W_{2} e \cdots W_{t} e W_{r}$, where $W_{i}$ is a $(v, u)$-walk in $G$ for each $1 \leq i \leq t$, and $W_{r}$ is a $(v, v)$-walk in $H$. Here, $W_{r}$ either contains no $e$, or contains no $u e v$, or veu appears earlier than uev. Without loss of generality, we suppose veu appears earlier than uev in $W_{r}$. Then $W_{r}$ can be decomposed to $W_{r}=W_{t+1} e W_{t+2}$ such that $W_{t+1} \in W_{k_{t+1}}(G ; v)$ for some $k_{t+1} \geq 0$ and $W_{t+2}$ is a $(u, v)$-walk in $H$. In this case, we define $h_{k}(W)=\overleftarrow{W_{1}} e \overleftarrow{W_{2}} e \cdots \overleftarrow{W_{t}} e f_{k_{t+1}}\left(W_{t+1}\right) e \overleftarrow{W_{t+2}}$. Then $h_{k}(W) \in W_{k}(H ; u,[e])$. Now it is easy to show that the map $h_{k}: W_{k}(H ; v,[e]) \leq W_{k}(H ; u,[e])$ defined as above is an injection. Therefore, $M_{k}(H ; v,[e]) \leq M_{k}(H ; u,[e])$.

Moreover, if $(G ; v) \prec(G ; u)$, then $M_{k_{0}}(G ; v)<M_{k_{0}}(G ; u)$ for some $k_{0}>0$. Thus, $M_{k_{0}}(H ; v)<M_{k_{0}}(H ; u)$ by $(2)$, which implies $(H ; v) \prec(H ; u)$. 
Lemma 6. Let $G$ be a graph and $P=v_{0} v_{1} \cdots v_{m}$ be a path in $G$ such that $d_{G}\left(v_{0}\right)=1$. Let $q$ and $l$ be two nonnegative integers such that $0 \leq q<l$ and $q+l \leq m$. Suppose $v=v_{q}$ and $u=v_{l}$ are two vertices in $P$ such that $d_{G}\left(v_{i}\right)=2$ for each $0<i<\frac{q+l}{2}$. Let $a=\left\lfloor\frac{q+l}{2}\right\rfloor$. Then

(1) $(G ; v) \preceq(G ; u)$;

(2) If $q+l<m$, or $q+l=m$ and the condition $\mathcal{C}$ does not hold, then $(G ; v) \prec(G ; u)$, where $\mathcal{C}$ is: $d_{G}\left(v_{m}\right)=1$ and $d_{G}\left(v_{i}\right)=2$ for each $a+1 \leq i \leq m-1$.

(3) If $q+l$ is even, then $(G ; w, v) \preceq(G ; w, u)$ for each $w \in V(G) \backslash\left\{v_{0}, v_{1}, \ldots, v_{a-1}\right\}$.

Proof of Lemma 6. Let $e_{i}=v_{i} v_{i+1}$ for each $0 \leq i \leq m-1$. For each walk $W$ in $G\left[\left\{v_{0}, v_{1}\right.\right.$, $\left.\left.\ldots, v_{p+l}\right\}\right]$, denote by $\bar{W}$ the walk obtained from $\bar{W}$ by replacing each vertex $v_{x}$ with $v_{x^{\prime}}$ and the corresponding edges, where $x^{\prime}=q+l-x$. We distinguish the following two cases: Case $1 . q+l$ is even.

Let $k \geq 0$ and $W \in W_{k}(G ; v)$. Then $v_{a}=v_{\frac{q+l}{2}}$ has the same distance from $v$ and $u$ in $P$. If $W$ contains $v_{a}$ more than once, then it can be decomposed uniquely to $W=W_{1} W_{2} W_{3}$, such that $W_{2} \in W_{k_{2}}\left(G ; v_{a}\right)$ which is as long as possible, $W_{1}$ is a $\left(v, v_{a}\right)$-walk in $G$, and $W_{3}$ is a $\left(v_{a}, v\right)$-walk in $G$. In this case, let $f_{k}^{(1)}(W)=\overline{W_{1}} W_{2} \overline{W_{3}}$. Then $f_{k}^{(1)}(W) \in W_{k}(G ; u)$. If $W$ contains $v_{a}$ at most once, then let $f_{k}^{(1)}(W)=\bar{W}$. Obviously, the map $f_{k}^{(1)}: W_{k}(G ; v) \longrightarrow$ $W_{k}(G ; u)$ defined as above is an injection. Since $k$ is arbitrary, we have $(G ; v) \preceq(G ; u)$.

If $q+l<m$, then $f_{k}^{(1)}$ does not cover the walk $v_{l} v_{l+1} \cdots v_{m-1} v_{m} v_{m-1} \cdots v_{l+1} v_{l}$.

Now suppose $q+l=m$ and the condition $\mathcal{C}$ does not hold. Without loss of generality, suppose there exists some $a+1 \leq j \leq m-1$ with $d_{G}\left(v_{j}\right) \neq 2$. Then there exists a vertex $s \neq v_{j-1}, v_{j+1}$ such that $v_{j} s \in E(G)$. If $a+1 \leq j \leq l-1$, then $f_{k}^{(1)}$ does not cover the walk $v_{l} v_{l-1} \cdots v_{j-1} v_{j} s v_{j} \cdots v_{l-1} v_{l}$. If $l \leq j \leq m-1$, then $f_{k}^{(1)}$ does not cover the walk $v_{l} v_{l+1} \cdots v_{j} s v_{j} \cdots v_{l+1} v_{l}$. Therefore, if $q+l<m$, or $q+l=m$ and the condition $\mathcal{C}$ does not hold, then $M_{k_{0}}(G ; v)<M_{k_{0}}(G ; u)$ for some $k_{0} \geq 0$. This implies Lemma 6 (2) holds.

Let $w \in V(G) \backslash\left\{v_{0}, v_{1}, \ldots, v_{a-1}\right\}$ and $W \in W_{k}(G ; w, v)$. Then $W$ must contain $v_{a}$. Thus, $W$ can be decomposed uniquely to $W=W_{1} W_{2}$ such that $W_{1} \in W_{k_{0}}\left(G ; w, v_{a}\right)$ which is as long as possible and $W_{2}$ is a $\left(v_{a}, v\right)$-walk in $G$. Then the map $g_{k}: W_{k}(G ; w, v) \rightarrow W_{k}(G ; w, u)$ defined as $g_{k}(W)=W_{1} \overline{W_{2}}$ is an injection. Therefore, $(G ; w, v) \preceq(G ; w, u)$.

Case 2. $q+l$ is odd.

Let $k \geq 0, W \in W_{k}(G ; v)$, and $e_{a}=v_{a} v_{a+1}$. If $W$ contains $e_{a}$, then it must contain $e_{a}$ at least twice and can be decomposed uniquely to $W=W_{1} W_{2} e_{a} W_{3} e_{a} W_{4}$, such that $W_{1} \in W_{k_{1}}\left(G ; v, v_{a}\right)$, which contains $v_{a}$ only once; $W_{2} \in W_{k_{2}}\left(G ; v_{a}\right)$, which does not contain $e_{a} ; W_{3} \in W_{k_{3}}\left(G ; v_{a+1}\right)$, which is as long as possible; and $W_{4} \in W_{k_{4}}\left(G ; v_{a}, v\right)$, which does not contain $e_{a}$. In this case, let $f_{k}^{(2)}(W)=\overline{W_{1}} W_{3} e_{a} \overline{W_{2}} e_{a} \overline{W_{4}}$. If $W$ does not contain $e_{a}$, let $f_{k}^{(2)}(W)=\bar{W}$. Then the $\operatorname{map} f_{k}^{(2)}: W_{k}(G ; v) \longrightarrow W_{k}(G ; u)$ defined as above is an injection. Thus, $(G ; v) \preceq(G ; u)$.

The proof of (Case 2) when $q+l$ is odd is the same as that of Case 1.

Remark 1. Lemma 6 (3) does not hold if $q+l$ is odd. For example, let $G$ be the graph obtained from $P_{8}=v_{0} v_{1} \cdots v_{7}$ and a new vertex $w$ by adding the edge $v_{3} w$. Let $v=v_{1}$ and $u=v_{4}$. Then $M_{3}(G ; w, v) \geq 1$ and $M_{3}(G ; w, u)=0$. Thus, $(G ; w, v) \preceq(G ; w, u)$ does not hold in $G$.

\section{Graphs with the Maximum Estrada Index in $\mathcal{U}(n, d)$}

In this section, we determine the graphs with the maximum Estrada index among $\mathcal{U}(n, d)$.

Let $C_{t}\left(n_{1}, n_{2}, \ldots, n_{t}\right)$ be the graph obtained from the cycle $C_{t}=v_{1} v_{2} \cdots v_{t} v_{1}$ by attaching $n_{i}$ vertices to $v_{i}$ for $i=1,2, \ldots, l$. Let $C_{n}^{*} \cong C_{n-1}(1,0, \ldots, 0)$ and $X_{n} \cong C_{3}(0,0, n-3)$. Let $\mathcal{U}(n)$ be the set of all unicyclc graphs of order $n$.

The following theorem characterizes the graphs with greatest, second-greatest, smallest, and second-smallest Estrada indices among the unicyclic graphs in $\mathcal{U}(n)$. 
Theorem 2 ([33]). Among the unicyclic graphs in $\mathcal{U}(n)$,

(i) According to references [22,34], the cycle $C_{n}$ has smalles tindex and the graph $C_{n}^{*}$ has secondsmallest Estrada index;

(ii) According to references [22,34], the graph $X_{n}$ has the greatest Estrada index;

(iii) The graph $C_{3}(0,1, n-4)$ has the second-greatest Estrada index.

For $G \in \mathcal{U}(n, d)$, we have $n \geq 3$ and $1 \leq d \leq n-2$. If $d=1$, then $G=C_{3}$. By Theorem 2, the graphs with the maximum Estrada indices among the graphs in $\mathcal{U}(n, 2)$ and $\mathcal{U}(n, 3)$ are $X_{n}$ and $C_{3}(0,1, n-4)$, respectively. Therefore, we assume $d \geq 4$ and $n \geq 6$ in the following. Now we give some lemmas.

Lemma 7. Let $P=v_{0} v_{1} \cdots v_{d-1} v_{d}$ be a path, where $d \geq 4$ is even. Let $H$ be the graph obtained from $P$ and a new vertex $v_{d+1}$ by adding the edges $v_{\frac{d}{2}-1} v_{d+1}$ and $v_{\frac{d}{2}} v_{d+1}$; see Figure 1 . Then $\left(H ; v_{\frac{d}{2}-1}\right) \prec\left(H ; v_{\frac{d}{2}}\right)$.

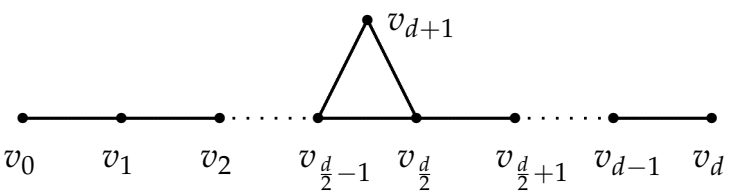

Figure 1. Graph $H$ in Lemma 7.

Proof of Lemma 7. Let $e_{i}=v_{i} v_{i+1}$ for each $0 \leq i \leq d-1$. Let $e=v_{\frac{d}{2}-1} v_{d+1}$ and $\bar{e}=$ $v_{\frac{d}{2}} v_{d+1}$. For each walk $W$ in $H\left[\left\{v_{0}, v_{1}, \ldots, v_{\frac{d}{2}-1}, v_{d+1}\right\}\right]$ and each $0 \leq i \leq \frac{d}{2}-1$, denote by $\bar{W}$ the walk obtained from $W$ by replacing $v_{i}$ with $v_{d-1-i}$ and the corresponding edges. Let $k \geq 0$ and $W \in W_{k}\left(H ; v_{\frac{d}{2}-1}\right)$. If $W$ does not contain $v_{\frac{d}{2}}$, then define $f_{k}(W)=\bar{W}$. If $W$ contains $v_{\frac{d}{2}}$, then $W$ can be decomposed uniquely to $W^{2}=W_{1} W_{2} W_{3} W_{4} W_{5}$, such that $W_{1} \in W_{k_{1}}\left(G ; v_{\frac{d}{2}-1}\right)$ for some $k_{1} \geq 0, W_{5} \in W_{k_{5}}\left(H ; v_{\frac{d}{2}-1}\right)$ for some $k_{5} \geq 0, W_{3} \in W_{k_{3}}\left(H ; v_{\frac{d}{2}}\right)$ which is as long as possible, $W_{2}=e_{\frac{d}{2}-1}$ or $e v_{d+1} \bar{e}, W_{4}=e_{\frac{d}{2}-1}$ or $\bar{e} v_{d+1} e$. Obviously, neither $W_{1}$ nor $W_{5}$ contains $v_{\frac{d}{2}}$. In this case, we define $f_{k}(W)=W_{3} W_{4} W_{5} W_{2} \overline{W_{1}}$. Then it is easy to show that the map $f_{k}: W_{k}\left(H ; v_{\frac{d}{2}-1}\right) \longrightarrow W_{k}\left(H ; v_{\frac{d}{2}}\right)$ defined as above is an injection. Since $f_{k}$ does not cover the walk $v_{\frac{d}{2}}^{2} v_{\frac{d}{2}+1} \cdots v_{d-1} v_{d}^{2} v_{d-1} \cdots v_{\frac{d}{2}+1} v_{\frac{d}{2}}$, we have $\left(H ; v_{\frac{d}{2}-1}\right) \prec\left(H ; v_{\frac{d}{2}}\right)$.

Lemma 8. Let $C_{4}=x v y u x$ be a 4-cycle. Denote by $H$ the graph obtained from $C_{4}$ by attaching two paths $P_{p}=v_{1} v_{2} \cdots v_{p}$ and $P_{q}=u_{1} u_{2} \cdots u_{q}$ at vertices $v$ and $u$, respectively; see Figure 2. If $0 \leq p<q$, then $(H ; v) \prec(H ; u)$.

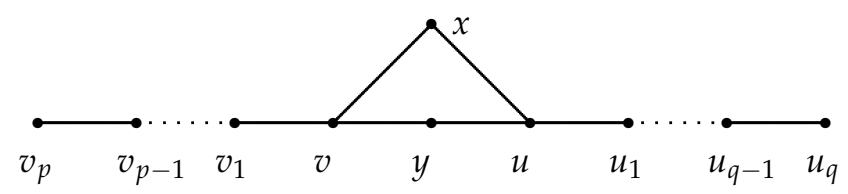

Figure 2. Graph $H$ in Lemmas 8 and 9.

Proof of Lemma 8. For each walk $W$ in $H\left[\left\{x, y, v, v_{1}, \ldots, v_{p}\right\}\right]$ and each $1 \leq i \leq p$, denote by $\bar{W}$ the walk obtained from $W$ by replacing $v_{i}$ with $u_{i}, v$ with $u$ and the corresponding edges. Let $k \geq 0$ and $W \in W_{k}(H ; v)$. If $W$ contains neither $x$ nor $y$, then let $f_{k}(W)=\bar{W}$. If $W$ contains $x$ or $y$, then $W$ can be decomposed uniquely to $W=W_{1} W_{2} W_{3}$, such that $W_{1}$ is a $(v, s)$-walk; $W_{2}$ is a $(s, t)$-walk which is as long as possible, where $s, t \in\{x, y\}$; and $W_{3}$ is a $(t, v)$-walk. It is obvious that both of $W_{1}$ and $W_{3}$ are walks in $H\left[\left\{x, y, v, v_{1}, \ldots, v_{p}\right\}\right]$. In this case, we define $f_{k}(W)=\overline{W_{1}} W_{2} \overline{W_{3}}$. Then it is easy to show 
that the map $f_{k}: W_{k}(H ; v) \longrightarrow W_{k}(H ; u)$ defined as above is an injection. Since $p<q, f_{k}$ does not cover the walk $u u_{1} \cdots u_{q-1} u_{q} u_{q-1} \cdots u_{1} u$. Therefore, we have $(H ; v) \prec(H ; u)$.

Lemma 9. Let $H$ be the graph depicted in Figure 2. If $0 \leq p \leq q$ and $q \geq 1$, then $(H ; y) \prec(H ; u)$.

Proof of Lemma 9. Let $k \geq 0$ be an arbitrary integer. By the definition of the walk, we have

$$
\begin{aligned}
M_{k}(H ; y) & =M_{k-1}(H ; y, u)+M_{k-1}(H ; y, v) \\
& =M_{k-1}(H ; u, y)+M_{k-1}(H ; y, v) \\
& =M_{k-1}(H ; u, y)+M_{k-1}(H ; x, v)
\end{aligned}
$$

and

$$
\begin{aligned}
M_{k}(H ; u) & =M_{k-1}(H ; u, y)+M_{k-1}(H ; u, x)+M_{k-1}\left(H ; u, u_{1}\right) \\
& =M_{k-1}(H ; u, y)+M_{k-1}(H ; x, u)+M_{k-1}\left(H ; u, u_{1}\right)
\end{aligned}
$$

Since $M_{k-1}\left(H ; u, u_{1}\right) \geq 0$, in order to prove $M_{k}(H ; y) \leq M_{k}(H ; u)$, it suffices to show $M_{k-1}(H ; x, v) \leq M_{k-1}(H ; x, u)$, i.e., $M_{k}(H ; x, v) \leq M_{k}(H ; x, u)$ for each $k \geq 0$. We prove this by induction on $k$.

If $k=1, M_{k}(H ; x, v)=M_{k}(H ; x, u)=1$. If $k=2, M_{k}(H ; x, v)=M_{k}(H ; x, u)=0$. Now suppose $k \geq 3$ and let $W \in W_{k}(H ; x, v)$. We consider the edge $e$ preceding the last vertex $v$ in $W$. If $e=(x, v)$, then $W$ can be written as $W=W_{1}(x, v) v$. In this case, let $f_{k}(W)=W_{1}(x, u) u$. If $e=(y, v)$, then $W$ can be written as $W=W_{1}(y, v) v$. In this case, let $f_{k}(W)=W_{1}(y, u) u$. If $e=v_{1} v$, then $W$ can be uniquely decomposed to $W_{1} W_{2}$, such that $W_{1} \in W_{k_{1}}(x, v)$ for some $0 \leq k_{1}<k$ which is as large as possible, and $W_{2} \in W_{k_{2}}(v, v)$ for some $k_{2} \geq 0$. Obviously, $W_{2}$ is a walk in $H\left[\left\{v, v_{1}, \ldots, v_{p}\right\}\right]$. Define $\overline{W_{2}}$ the walk obtained from $W_{2}$ by replacing $v_{i}$ with $u_{i}$ for each $1 \leq i \leq p, v$ with $u$, and the corresponding edges. By the inductive hypothesis, there is an injection $f_{k_{1}}: W_{k_{1}}(H ; x, v) \rightarrow W_{k_{1}}(H ; x, u)$. In this case, let $f_{k}(W)=f_{k_{1}}\left(W_{1}\right) \overline{W_{2}}$. Then it is easy to show that the map $f_{k}: W_{k}(H ; x, v) \longrightarrow$ $W_{k}(H ; x, u)$ defined as above is an injection. Therefore, $M_{k}(H ; x, v) \leq M_{k}(H ; x, u)$.

Since $q \geq 1, M_{2}(H ; y)=d_{H}(y)=2<3=d_{H}(u)=M_{2}(H ; u)$. Thus, $(H ; y) \prec(H ; u)$. This completes the proof.

Let $P=v_{0} v_{1} \cdots v_{d}$ be a path of length $d$ with $d \geq 2$. Let $\Delta_{n}^{d}$ be the graph obtained from $P$ and a new vertex $v_{d+1}$ by adding the edges $v_{\left\lfloor\frac{d}{2}\right\rfloor} v_{d+1}$ and $v_{\left\lfloor\frac{d}{2}\right\rfloor+1} v_{d+1}$, and attaching $n-d-2$ pendant edges at the vertex $v_{\left\lfloor\frac{d}{2}\right\rfloor}$ (see Figure 3 ).

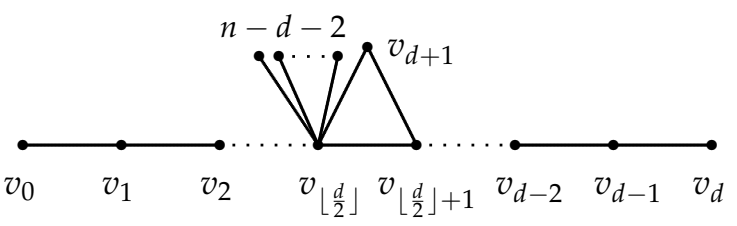

Figure 3. Graph $\Delta_{n}^{d}$.

Lemma 10. Let $P=v_{0} v_{1} \cdots v_{d}$ be a path of length $d \geq 4$. Let $G_{v_{k}, v}$ be the graph with diameter $d$ obtained from $P$ and a new vertex $v_{d+1}$ by adding the edges $v_{k} v_{d+1}$ and $v_{k+1} v_{d+1}$, and attaching $n-d-2$ pendant edges at one vertex $v \in V(P) \cup\left\{v_{d+1}\right\}$, where $0 \leq k \leq d-1$ and $n-d-2 \geq$ 0 . Then $\operatorname{EE}\left(G_{v_{k}, v}\right) \leq E E\left(\Delta_{n}^{d}\right)$, with equality if and only if $G_{v_{k}, v} \cong \Delta_{n}^{d}$, where $\Delta_{n}^{d}$ is depicted in Figure 3.

Proof of Lemma 10. Denote by $\mathcal{B}$ the set of all graphs $G_{v_{k}, v}$. Let $G^{*}$ be the graph in $\mathcal{B}$ with the maximum Estrada index. Then there exists some $0 \leq k \leq d-1$ such that $G^{*}$ is obtained from $P$ and $v_{d+1}$ by adding the edges $v_{k} v_{d+1}$ and $v_{k+1} v_{d+1}$, and attaching $n-d-2$ pendant edges at a vertex $v$ for some vertex $v \in V(P) \cup\left\{v_{d+1}\right\}$. We show that $v_{k}=v=v_{\left\lfloor\frac{d}{2}\right\rfloor}$, i.e., 
$G^{*} \cong \Delta_{n}^{d}$. For each vertex $v_{i}$ in $P$, let $\overline{N_{G^{*}}\left(v_{i}\right)}=N_{G^{*}}\left(v_{i}\right) \cap\left(V\left(G^{*}\right) \backslash V(P)\right)$. We distinguish the following two cases.

Case 1. $d$ is odd.

We show that for each $v_{i} \in V(P) \backslash\left\{v_{\left\lfloor\frac{d}{2}\right\rfloor}, v_{\left\lfloor\frac{d}{2}\right\rfloor+1}\right\}$, we have $\overline{N_{G^{*}}\left(v_{i}\right)}=\varnothing$.

Let $t$ be the minimum index with $\overline{N_{G^{*}}\left(v_{t}\right)} \neq \varnothing$. Suppose $\overline{N_{G^{*}}\left(v_{t}\right)}=\left\{w_{1}, w_{2}, \ldots, w_{s}\right\}$. If $t<\left\lfloor\frac{d}{2}\right\rfloor$, then $t+(t+2)<d$ and $t+(t+2)$ is even. Let $G_{0}=G^{*}-\left\{v_{t} w_{i} \mid 1 \leq i \leq s\right\}$. By Lemma $6,\left(G_{0} ; v_{t}\right) \prec\left(G_{0} ; v_{t+2}\right)$, and for each $1 \leq i \leq s,\left(G_{0} ; w_{i}, v_{t}\right) \preceq\left(G_{0} ; w_{i}, v_{t+2}\right)$. Define $G^{\prime}=G_{0}+\left\{v_{t+2} w_{i} \mid 1 \leq i \leq s\right\}$. Then $G^{\prime} \in \mathcal{B}$ and $E E\left(G^{*}\right)<E E\left(G^{\prime}\right)$ by Lemma 1, a contradiction to the choice of $G^{*}$. Therefore, $t \geq\left\lfloor\frac{d}{2}\right\rfloor$, i.e., $\overline{N_{G^{*}}\left(v_{i}\right)}=\varnothing$ for each $i<$ $\left\lfloor\frac{d}{2}\right\rfloor$. Similarly, we have $\overline{N_{G^{*}}\left(v_{i}\right)}=\varnothing$ for each $i>\left\lfloor\frac{d}{2}\right\rfloor+1$. Thus, $v_{k}=v_{\left\lfloor\frac{d}{2}\right\rfloor}$ and $v \in$ $\left\{v_{\left\lfloor\frac{d}{2}\right\rfloor}, v_{\left\lfloor\frac{d}{2}\right\rfloor+1}, v_{d+1}\right\}$.

Obviously, $G^{*} \cong \Delta_{n}^{d}$ if $n-d-2=0$. Now we suppose $n-d-2>0$. Then $d_{G^{*}}(v) \geq 3$. Suppose $v=v_{d+1}$. Let $G_{0}=G^{*}-v_{\left\lfloor\frac{d}{2}\right\rfloor-1} v_{\left\lfloor\frac{d}{2}\right\rfloor}$ and $G_{1}=G_{0}-v_{\left\lfloor\frac{d}{2}\right\rfloor} v_{d+1}$. Then $N_{G_{1}}\left(v_{\left\lfloor\frac{d}{2}\right\rfloor}\right) \subseteq N_{G_{1}}\left(v_{d+1}\right)$ and $d_{G_{1}}\left(v_{\left\lfloor\frac{d}{2}\right\rfloor}\right)=1<2 \leq d_{G_{1}}\left(v_{d+1}\right)$. Thus, we have $\left(G_{1} ; v_{\left\lfloor\frac{d}{2}\right\rfloor}\right) \prec\left(G_{1} ; v_{d+1}\right)$ by Lemma 4 and $\left(G_{0} ; v_{\left\lfloor\frac{d}{2}\right\rfloor}\right) \prec\left(G_{0} ; v_{d+1}\right)$ by Lemma 5 . Note that for each $k \geq 0, M_{k}\left(G_{0} ; v_{\left\lfloor\frac{d}{2}\right\rfloor-1}, v_{\left\lfloor\frac{d}{2}\right\rfloor}\right)=M_{k}\left(G_{0} ; v_{\left\lfloor\frac{d}{2}\right\rfloor-1}, v_{d+1}\right)=0$. By Lemma 1 , we get $E E\left(G^{*}\right)<E E\left(\Delta_{n}^{d}\right)$, a contradiction to the choice of $G^{*}$. Therefore, $v \neq v_{d+1}$, i.e., $G^{*} \cong \Delta_{n}^{d}$.

Case 2. $d$ is even.

By an argument similar to that of Case 1 , we have $\overline{N_{G^{*}}\left(v_{i}\right)}=\varnothing$ for each $i<\left\lfloor\frac{d}{2}\right\rfloor-1$ and $i>\left\lfloor\frac{d}{2}\right\rfloor+1$. Thus, $v_{k}=v_{\left\lfloor\frac{1}{2}\right\rfloor-1}$ or $v_{\left\lfloor\frac{d}{2}\right\rfloor}$, and $v \in\left\{v_{\left\lfloor\frac{d}{2}\right\rfloor-1}, v_{\left\lfloor\frac{d}{2}\right\rfloor}, v_{\left\lfloor\frac{d}{2}\right\rfloor+1}, v_{d+1}\right\}$. Without loss of generality, we may suppose $v_{k}=v_{\left\lfloor\frac{d}{2}\right\rfloor}$. Then $G^{*} \cong \Delta_{n}^{d}$ if $n-d-2=0$. Now suppose $n-d-2>0$. By an argument similar to that in Case 1 , we have $v \neq v_{d+1}$. Suppose $v=v_{\left\lfloor\frac{d}{2}\right\rfloor-1}$ or $v_{\left\lfloor\frac{d}{2}\right\rfloor+1}$. Let $\left\{u_{1}, u_{2}, \ldots, u_{n-d-2}\right\}$ be the set of all pendant vertices adjacent to $v$ and $G_{0}=G^{*}-\left\{v u_{i} \mid 1 \leq i \leq n-d-2\right\}$. Then by Lemmas 6 and $7,\left(G_{0} ; v\right) \prec\left(G_{0} ; v_{\left\lfloor\frac{d}{2}\right\rfloor}\right)$, and for each $1 \leq i \leq n-d-2,\left(G_{0} ; u_{i}, v\right) \preceq\left(G_{0} ; u_{i}, v_{\left\lfloor\frac{d}{2}\right\rfloor}\right)$. By Lemma $1, E E\left(G^{*}\right)<E E\left(\Delta_{n}^{d}\right)$, a contradiction to the choice of $G^{*}$. Therefore, $v=v_{\left\lfloor\frac{d}{2}\right\rfloor}$, i.e., $G^{*} \cong \Delta_{n}^{d}$. This completes the proof.

Lemma 11. Let $P=v_{0} v_{1} \cdots v_{d}$ be a path of length $d \geq 4$. Let $G_{k, t}$ be the graph with diameter $d$ obtained from $P$ and a new vertex $v_{d+1}$ by adding the edges $v_{k} v_{d+1}$ and $v_{k+2} v_{d+1}$, and attaching $n-d-2$ pendant edges at $v_{t}$, where $0 \leq k \leq d-2,1 \leq t \leq d-1$ and $n-d-2 \geq 0$. Let $G_{1}=G_{\left\lfloor\frac{d}{2}\right\rfloor,\left\lfloor\frac{d}{2}\right\rfloor}$ and $G_{2}=G_{\left\lfloor\frac{d}{2}\right\rfloor-1,\left\lfloor\frac{d}{2}\right\rfloor-1}$ be depicted in Figure 4 .

(i) If $d$ is odd, then $E E\left(G_{k, t}\right) \leq E E\left(G_{1}\right)$, with equality if and only if $G_{k, t} \cong G_{1}$.

(ii) If $d$ is even, then $E E\left(G_{k, t}\right) \leq \max \left\{E E\left(G_{1}\right), E E\left(G_{2}\right)\right\}$, with equality if and only if $G_{k, t}$ is isomorphic to the graph with a larger Estrada index between $G_{1}$ and $G_{2}$.

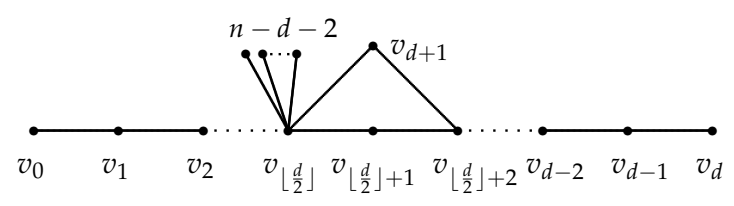

$G_{1}$

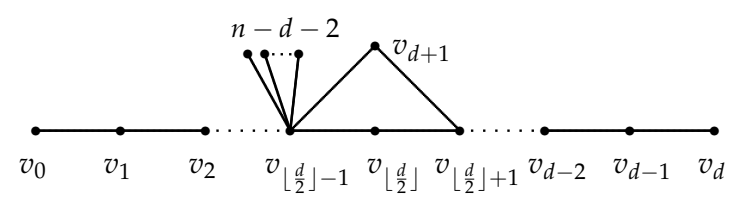

$G_{2}$

Figure 4. Graphs $G_{1}$ and $G_{2}$. 
Proof of Lemma 11. Denote by $\mathcal{B}$ the set of all graphs $G_{k, t}$. Let $G^{*}$ be the graph in $\mathcal{B}$ with the maximum Estrada index. Then there exists some $0 \leq k \leq d-2$ and $1 \leq t \leq d-1$ such that $G^{*}$ is obtained from $P$ and $v_{d+1}$ by adding the edges $v_{k} v_{d+1}$ and $v_{k+2} v_{d+1}$, and attaching $n-d-2$ pendant edges at the vertex $v_{t}$. We distinguish the following two cases.

Case $1 . d$ is odd.

Without loss of generality, we suppose $n-d-2>0$. Let $\left\{u_{1}, u_{2}, \ldots, u_{n-d-2}\right\}$ be the set of all pendant vertices adjacent to $v_{t}$. Let $H=G^{*}-\left\{v_{t} u_{i} \mid 1 \leq i \leq n-d-2\right\}$. We show in the following that either $k=t-2=\left\lfloor\frac{d}{2}\right\rfloor-1$, or $k=t=\left\lfloor\frac{d}{2}\right\rfloor$.

Suppose $k<\left\lfloor\frac{d}{2}\right\rfloor-1$. Then $k+(k+4)<d$ and $k+(k+4)$ is even. Moreover, $\left(H ; v_{i}\right) \prec\left(H ; v_{k}\right)$ for each $1 \leq i<k$ by Lemma $6,\left(H ; v_{k}\right) \prec\left(H ; v_{k+2}\right)$ and $\left(H ; v_{k+1}\right) \prec$ $\left(H_{1} ; v_{k+2}\right)$ by Lemmas 8 and 9 . Thus, $t \geq k+2$ by Lemma 1 . Now let $G^{\prime}=G^{*}-v_{k} v_{d+1}+$ $v_{k+4} v_{d+1}$. Then $G^{\prime} \in \mathcal{B}$ and $E E\left(G^{*}\right)<E E\left(G^{\prime}\right)$ by Lemmas 1 and 6 , a contradiction to the choice of $G^{*}$. Therefore, $k \geq\left\lfloor\frac{d}{2}\right\rfloor-1$. Similarly, $k \leq\left\lfloor\frac{d}{2}\right\rfloor$. Thus, $k=\left\lfloor\frac{d}{2}\right\rfloor-1$ or $\left\lfloor\frac{d}{2}\right\rfloor$. Suppose $k=\left\lfloor\frac{d}{2}\right\rfloor-1$. Then $\left(H ; v_{i}\right) \prec\left(H ; v_{\left\lfloor\frac{d}{2}\right\rfloor+1}\right)$ for each $i \neq\left\lfloor\frac{d}{2}\right\rfloor+1$ with $1 \leq i \leq d-1$ by Lemmas 6,8 , and 9. Thus, $t=\left\lfloor\frac{d}{2}\right\rfloor+1$ from the choice of $G^{*}$. Similarly, $t=\left\lfloor\frac{d}{2}\right\rfloor$ if $k=\left\lfloor\frac{d}{2}\right\rfloor$. Since $d$ is odd, we get $G^{*} \cong G_{1}$.

Case 2. $d$ is even.

By a similar argument to that in Case 1, we can show that either $k=t-2=\left\lfloor\frac{d}{2}\right\rfloor-2$, or $k=t-2=\left\lfloor\frac{d}{2}\right\rfloor-1$, or $k=t=\left\lfloor\frac{d}{2}\right\rfloor-1$, or $k=t=\left\lfloor\frac{d}{2}\right\rfloor$. Note that $G^{*}$ has a maximum among $\mathcal{B}$. Therefore, $G^{*}$ is isomorphic to the graph between $G_{1}$ and $G_{2}$ with a larger Estrada index.

Now we give our main results.

Theorem 3. Let $G$ be the graph with the maximum Estrada index among $\mathcal{U}(n, d)$. Let $\Delta_{n}^{d}, G_{1}$ and $G_{2}$ be depicted in Figures 3 and 4 . Then $G \in\left\{\Delta_{n}^{d}, G_{1}\right\}$ if $d$ is odd, and $G \in\left\{\Delta_{n}^{d}, G_{1}, G_{2}\right\}$ otherwise.

Proof of Theorem 3. If $d=1$, then $G \cong C_{3}$. If $d=2$ or 3 , then $G \cong \Delta_{n}^{d}$ by Theorem 2 . Thus, the result holds when $d \leq 3$. We assume $n-2 \geq d \geq 4$ below.

By Theorem 2, $G \neq C_{n}$. Let $P_{d}=v_{0} v_{1} \cdots v_{d}$ be an induced path of length $d$ and $C_{q}$ be the unique cycle in $G$. Since $G \nsubseteq C_{n}, \min \left\{d\left(v_{0}\right), d\left(v_{d}\right)\right\}=1$-say, $d\left(v_{0}\right)=1$. Thus, we can make some claims.

Claim 1. $V\left(P_{d}\right) \cap V\left(C_{q}\right) \neq \varnothing$.

Proof of Claim 1. Otherwise, since $G$ is connected, there exists a shortest path $Q=$ $v_{i} u_{k} u_{k+1} \cdots u_{l-1} u_{l}$ connecting $C_{q}$ and $P_{d}$, where $u_{l} \in V\left(C_{q}\right)$ and $v_{i} \in V\left(P_{d}\right)$, and $u_{k}, u_{k+1}, \ldots$ and $u_{l-1} \in V(G) \backslash\left(V\left(C_{q}\right) \cup V\left(P_{d}\right)\right)$. Denote by $G_{1}$ and $G_{2}$ the connected components containing $v_{i}$ and $u_{k}$ in $G-v_{i} u_{k}$, respectively. Let $G^{\prime}$ be the graph obtained from $G_{1}$ and $G_{2}$ by identifying $v_{i}$ with $u_{k}$, and attaching a pendant vertex to the common vertex. Then $G^{\prime} \in \mathcal{U}_{n}^{d}$ and $E E(G)<E E\left(G^{\prime}\right)$ by Lemma 3, a contradiction.

By Claim 1, $V\left(C_{q}\right) \cap V\left(P_{d}\right) \neq \varnothing$. Denote $C_{q}=v_{k} v_{k+1} \cdots v_{l-1} v_{l} v_{d+1} v_{d+2} \cdots v_{s} v_{k}$, where $s \geq d+1,\left\{v_{k}, v_{k+1}, \ldots, v_{l-1}, v_{l}\right\}=V\left(C_{q}\right) \cap V\left(P_{d}\right)$ and $\left\{v_{d+1}, v_{d+2}, \ldots, v_{s}\right\}=$ $V\left(C_{q}\right) \backslash V\left(P_{d}\right)$. By a similar argument, we have

Claim 2. $d(v)=1$ for each vertex $v \in V(G) \backslash\left(V\left(C_{q}\right) \cup V\left(P_{d}\right)\right)$.

By Theorem 1, we have

Claim 3. All pendant vertices except $v_{0}$ and $v_{d}$ in $G$ are adjacent to one common vertex $v$.

Claim 4. $k \neq l$. 
Proof of Claim 4. Suppose $k=l$. Then $s \geq d+2$ and $k \neq 0, d$. Since $d \geq 4$, we can assume $k \geq 2$ (otherwise, relabel the vertices in $P_{d}$ ). Let $N_{G}\left(v_{d+1}\right) \backslash\left\{v_{k}, v_{d+2}\right\}=\left\{w_{1}, w_{2}, \ldots, w_{t}\right\}$, $H=G-\left\{v_{d+1} v_{d+2}\right\} \cup\left\{v_{d+1} w_{i} \mid 1 \leq i \leq t\right\}$ and $G^{\prime}=H+\left\{v_{k-1} v_{d+2}\right\} \cup\left\{v_{k-1} w_{i} \mid\right.$ $1 \leq i \leq t\}$. Then $N_{H}\left(v_{d+1}\right) \subseteq N_{H}\left(v_{k-1}\right), d_{H}\left(v_{d+1}\right)=1<d_{H}\left(v_{k-1}\right)$ and $G^{\prime} \in \mathcal{U}_{n}^{d}$. By Lemma 4 , we have $\left(H ; v_{d+1}\right) \prec\left(H ; v_{k-1}\right)$ and for each vertex $w \in\left\{v_{d+2}, w_{1}, w_{2}, \ldots, w_{t}\right\}$, $\left(H ; w, v_{d+1}\right) \preceq\left(H ; w, v_{k-1}\right)$. Thus, $E E(G)<E E\left(G^{\prime}\right)$ by Lemma 1 , a contradiction.

Claim 5. If $l=k+1$, then $s=d+1$; and if $l \geq k+2$, then $l=k+2$ and $s=d+1$.

Proof of Claim 5. Suppose $l=k+1$. If $s \geq d+3$, by letting $N_{G}\left(v_{d+1}\right) \backslash\left\{v_{k+1}, v_{d+2}\right\}=$ $\left\{w_{1}, w_{2}, \ldots, w_{t}\right\}, H=G-\left\{v_{d+1} v_{d+2}\right\} \cup\left\{v_{d+1} w_{i} \mid 1 \leq i \leq t\right\}$ and $G^{\prime}=H+\left\{v_{k} v_{d+2}\right\} \cup$ $\left\{v_{k} w_{i} \mid 1 \leq i \leq t\right\}$, then $N_{H}\left(v_{d+1}\right) \subseteq N_{H}\left(v_{k}\right), d_{H}\left(v_{d+1}\right)=1<d_{H}\left(v_{k}\right)$ and $G^{\prime} \in$ $\mathcal{U}_{n}^{d}$. Moreover, $\left(H ; v_{d+1}\right) \prec\left(H ; v_{k}\right)$ and $\left(H ; w, v_{d+1}\right) \preceq\left(H ; w, v_{k}\right)$ for each vertex $w \in$ $\left\{v_{d+2}, w_{1}, w_{2}, \cdots, w_{t}\right\}$ by Lemma 4 . Thus, $E E(G)<E E\left(G^{\prime}\right)$ by Lemma 1 , a contradiction. Hence, $s=d+2$ or $d+1$.

Suppose $s=d+2$ when $l=k+1$. Since $d \geq 4$, we can assume $k \geq 2$ (otherwise, relabel the vertices in $\left.P_{d}\right)$. Let $N_{G}\left(v_{d+2}\right) \backslash\left\{v_{k}, v_{d+1}\right\}=\left\{w_{1}, w_{2}, \ldots, w_{t}\right\}, H=$ $G-\left\{v_{d+2} v_{d+1}\right\} \cup\left\{v_{d+2} w_{i} \mid 1 \leq i \leq t\right\}$ and $G^{\prime}=H+\left\{v_{k-1} v_{d+1}\right\} \cup\left\{v_{k-1} w_{i} \mid 1 \leq i \leq t\right\}$. Then $N_{H}\left(v_{d+2}\right) \subseteq N_{H}\left(v_{k-1}\right), d_{H}\left(v_{d+2}\right)=1<d_{H}\left(v_{k-1}\right)$ and $G^{\prime} \in \mathcal{U}_{n}^{d}$. By Lemma 4, $\left(H ; v_{d+2}\right) \prec\left(H ; v_{k-1}\right)$ and for each vertex $w \in\left\{v_{d+1}, w_{1}, w_{2}, \ldots, w_{t}\right\},\left(H ; w, v_{d+2}\right) \preceq$ $\left(H ; w, v_{k-1}\right)$. Thus, $E E(G)<E E\left(G^{\prime}\right)$ by Lemma 1 , a contradiction. This implies $s=d+1$ if $l=k+1$.

Now suppose $l \geq k+2$. Suppose $s \geq d+2$. Let $N_{G}\left(v_{s}\right) \backslash\left\{v_{k}, v_{s-1}\right\}=\left\{w_{1}, \ldots, w_{t}\right\}$, $H=G-\left\{v_{s} v_{s-1}\right\} \cup\left\{v_{s} w_{i} \mid 1 \leq i \leq t\right\}$ and $G^{\prime}=H+\left\{v_{k+1} v_{s-1}\right\} \cup\left\{v_{k+1} w_{i} \mid 1 \leq i \leq t\right\}$. Then $N_{H}\left(v_{s}\right) \subseteq N_{H}\left(v_{k+1}\right), d_{H}\left(v_{s}\right)=1<d_{H}\left(v_{k+1}\right)$ and $G^{\prime} \in \mathcal{U}_{n}^{d}$. By Lemma $4,\left(H ; v_{s}\right) \prec$ $\left(H ; v_{k+1}\right)$ and for each vertex $w \in\left\{v_{s-1}, w_{1}, w_{2}, \ldots, w_{t}\right\},\left(H ; w, v_{s}\right) \preceq\left(H ; w, v_{k+1}\right)$. Thus, $E E(G)<E E\left(G^{\prime}\right)$ by Lemma 1, a contradiction. Therefore, $s=d+1$. Since $s-d+1 \geq l-k$, we have $l=k+2$.

By Claims 5 and 3, if $l=k+1$, then $G$ is the unicyclic graph with maximum Estrada index of diameter $d$ obtained from $P_{d}$ and $v_{d+1}$ by adding the edges $v_{k} v_{d+1}$ and $v_{k+1} v_{d+1}$, and attaching $n-d-2$ pendant edges at one vertex $v \in V(P) \cup\left\{v_{d+1}\right\}$ for some $1 \leq k \leq$ $d-1$. By Lemma 10, we get

Claim 6. If $l=k+1$, then $G \cong \Delta_{n}^{d}$.

By Claims 5 and 3, if $l=k+1$, then $G$ is the unicyclic graph with the maximum Estrada index of diameter $d$ obtained from $P_{d}$ and $v_{d+1}$ by adding the edges $v_{k} v_{d+1}$ and $v_{k+2} v_{d+1}$, and attaching $n-d-2$ pendant edges at one vertex $v \in V(P)$ for some $1 \leq k \leq d-2$. By Lemma 11, we get

Claim 7. If $l=k+2$, then $G \cong G_{1}$ if $d$ is odd, and $G \in\left\{G_{1}, G_{2}\right\}$ if $d$ is even.

Now the proof is complete.

By Theorem 3, we can easily obtain the following corollary.

Corollary 1. Let $G$ be a graph in $\mathcal{U}(n, d)$. If the girth of $G$ is odd, then $E E(G) \leq E E\left(\Delta_{n}^{d}\right)$, with equality if and only if $G \cong \Delta_{n}^{d}$.

Liu et al. in [35] showed the following result on the spectral radii of unicyclic graphs.

Theorem 4 ([35]). Let $G$ be a graph in $\mathcal{U}(n, d), d \geq 1$. Then $\rho(G) \leq \rho\left(\Delta_{n}^{d}\right)$, and equality holds if and only if $G \cong \Delta_{n}^{d}$. 
Based on Theorems 3 and 4 and previous results on extremal values of Estrada index and spectral radius, we propose the following hypothesis.

Hypothesis 1. Let $G$ be a graph in $\mathcal{U}(n, d)$. Then $E E(G) \leq E E\left(\Delta_{n}^{d}\right)$, with equality if and only if $G \cong \Delta_{n}^{d}$.

Remark 2. To prove Hypothesis 1, it suffices to show that $E E\left(\Delta_{n}^{d}\right)>E E\left(G_{1}\right)$ and $E E\left(\Delta_{n}^{d}\right)>$ $E E\left(G_{2}\right)$ by Theorem 3. To show this, by previous methods and (1), it suffices to show that for $i=1,2$, the inequality $M_{k}\left(\Delta_{n}^{d}\right) \geq M_{k}\left(G_{i}\right)$ holds for each $k \geq 0$ and is strict for some $k_{0}>0$. However, this can not happen since $M_{4}\left(\Delta_{n}^{d}\right)=2 \sum_{j=1}^{n} d_{\Delta_{n}^{d}}\left(v_{j}\right)^{2}-2 m=2 \sum_{j=1}^{n} d_{G_{i}}\left(v_{j}\right)^{2}-2 m<$ $2 \sum_{j=1}^{n} d_{G_{i}}\left(v_{j}\right)^{2}-2 m+8=M_{4}\left(G_{i}\right)$ for $i=1,2$. Notice that $G_{1}$ and $G_{2}$ are both bipartite graphs. The hypothesis is true if we can show that for $i=1,2, \frac{M_{2 k-1}\left(\Delta_{n}^{d}\right)}{(2 k-1) !}+\frac{M_{2 k}\left(\Delta_{n}^{d}\right)}{(2 k) !} \geq \frac{M_{2 k}\left(G_{i}\right)}{(2 k) !}$ holds for all $k>0$ and is strict for some $k_{0}>0$.

\section{Conclusions}

In [1], Estrada proposed a graph invariant (the Estrada index) based on a Taylor series expansion of spectral moments. In this paper, we gave some transformations that can be used to compare the Estrada indices of two graphs. As applications, we determined the graphs with the maximum Estrada indices among all unicyclic graphs with fixed diameter $d$. We showed two candidate extremal graphs if $d$ is odd and three candidate extremal graphs if $d$ is even. For future research, it would be interesting to study Hypothesis 1.

Author Contributions: Methodology, W.N. and K.W.; investigation, W.N. and K.W.; writingoriginal draft preparation, K.W.; writing - review and editing, W.N.; funding acquisition, W.N. All authors have read and agreed to the published version of the manuscript.

Funding: This study was supported by the National Natural Science Foundation of China (number 11801568).

Institutional Review Board Statement: Not applicable.

Informed Consent Statement: Not applicable.

Data Availability Statement: Not applicable.

Conflicts of Interest: The authors declare no conflict of interest.

\section{References}

1. Estrada, E. Characterization of 3D molecular structure. Chem. Phys. Lett. 2000, 319, 713-718. [CrossRef]

2. Estrada, E.; Rodríguez-Valázquez, J.A. Subgraph centrality in complex networks. Phys. Rev. E 2005, 71, 056103. [CrossRef] [PubMed]

3. Estrada, E.; Rodríguez-Valázquez, J.A. Spectral measures of bipartivity in complex networks. Phys. Rev. E 2005, 72, 046105. [CrossRef] [PubMed]

4. Estrada, E.; Rodríguez-Valázquez, J.A.; Randić, M. Atomic branching in molecules. Int. J. Quantum Chem. 2006, 106, 823-832. [CrossRef]

5. Hayat, S.; Imran, M.; Liu, J. Correlation between the Estrada index and $\pi$-electronic energies for benzenoid hydrocarbons with applications to boron nanotubes. Int. J. Quantum Chem. 2019, 119, e26016. [CrossRef]

6. Du, Z. An edge grafting theorem on the Estrada index of graphs and its applications. Discret. Appl. Math. 2013, 161, 134-139. [CrossRef]

7. Shang, Y. Estrada and L-Estrada Indices of Edge-Independent Random Graphs. Symmetry 2015, 7, 1455-1462. [CrossRef]

8. Rodríguez, J. A note on new bounds for the Estrada Index. Linear Algebra Appl. 2019, 580, 121-127. [CrossRef]

9. Carmona, J.R.; Rodríguez, J. An increasing sequence of lower bounds for the Estrada index of graphs and matrices. Linear Algebra Appl. 2019, 580, 200-211. [CrossRef]

10. Shang, Y. Estrada Index and Laplacian Estrada Index of Random Interdependent Graphs. Mathematics 2020, 8, 1063. [CrossRef]

11. Rodríguez, J.; Aguayo, J.L.; Carmona, J.R.; Jahanbani, A. A note lower bounds for the Estrada index. Discret. Math. 2021, 344, 112303. [CrossRef] 
12. Rashid, M.A.; Ahmad, S.; Siddiqui, M.K.; Jahanbani, A.; Sheikholeslami, S.M.; Shao, Z. New Bounds for the Estrada Index of Phenylenes. Polycycl. Aromat Comp. 2020, 1-17. [CrossRef]

13. Brualdi, R.A.; Solheid, E.S. On the spectral radius of complementary acyclic matrices of zero and ones. SIAM J. Algebr. Discret. Methods 1986, 7, 265-272. [CrossRef]

14. He, S.; Li, S. On the signless Laplacian index of unicyclic graphs with fixed diameter. Linear Algebra Appl. 2012, 436, 252-261. [CrossRef]

15. Sun, Q.; Ikica, B.; Škrekovski, R.; Vukašinović, V. Graphs with a given diameter that maximize the Wiener index. Appl. Math. Comput. 2019, 356, 438-448.

16. Vukićević, Z.K.; Vujošević, S.; Popivoda, G. Unicyclic graphs with extremal values of arithmetic-geometric index. Discret. Appl. Math. 2021, 302, 67-75. [CrossRef]

17. Deng, H. A proof of a conjecture on the Estrada index. MATCH Commun. Math. Comput. Chem. 2009, 62, 599-606.

18. Zhai, C.; Wang, W. Minimal Estrada indices of the trees with a perfect matching. Electron. J. Linear Algebra 2016, 31, 134-146. [CrossRef]

19. Zhang, J.; Zhou, B.; Li, J. On Estrada index of trees. Linear Algebra Appl. 2011, 434, 215-223. [CrossRef]

20. Ilić, A.; Stevanović, D. The Estrada index of chemical trees. J. Math. Chem. 2010, 47, 305-314. [CrossRef]

21. Du, Z.; Zhou, B. The Estrada index of trees. Linear Algebra Appl. 2011, 435, 2462-2467. [CrossRef]

22. Du, Z.; Zhou, B. The Estrada index of unicyclic graphs. Linear Algebra Appl. 2012, 436, 3149-3159. [CrossRef]

23. Du, Z.; Zhou, B.; Xing, R. On maximum Estrada indices of graphs with given parameters. Linear Algebra Appl. 2012, 436, 3767-3772. [CrossRef]

24. Wang, L.; Fan, Y.; Wang, Y. Maximum Estrada index of bicyclic graphs. Discret. Appl. Math. 2015, 180, 194-199. [CrossRef]

25. Zhu, Z.; Tan, L.; Qiu, Z. Tricyclic graph with maximal Estrada index. Discret. Appl. Math. 2014, 162, 364-372. [CrossRef]

26. Andrade, E.; Lenes, E.; Mallea-Zepeda, E.; Robbiano, M.; Rodríguez, J. Extremal graphs for Estrada indices. Linear Algebra Appl. 2020, 588, 54-73. [CrossRef]

27. Wang, W.; Xu, W. Graphs with the maximal Estrada indices. Linear Algebra Appl. 2014, 446, 314-328. [CrossRef]

28. Du, Z.; Liu, Z. On the Estrada and Laplacian Estrada indices of graphs. Linear Algebra Appl. 2011, 435, 2065-2076. [CrossRef]

29. Zhu, Z. Maximal Estrada index of unicyclic graphs with perfect matching. J. Appl. Math. Comput. 2017, 54, 381-393. [CrossRef]

30. Liu, C.; Pan, Y.; Li, J. On the geometric-arithmetic Estrada index of graphs. Appl. Math. Comput. 2021, 391, 125700. [CrossRef]

31. Cvetković, D.; Doob, M.; Sachs, H. Spectra of Graphs-Theory and Application; Academic Press: New York, NY, USA, 1980.

32. Du, Z.; Zhou, B. On the Estrada index of graphs with given number of cut edges. Electron. J. Linear Algebra 2011, $22,586-592$. [CrossRef]

33. Zhu, Z. Some extremal properties of the resolvent energy, Estrada and resolvent Estrada indices of graphs. J. Math. Anal. Appl. 2017, 447, 957-970. [CrossRef]

34. Allem, L.; Capaverde, J.; Trevisan, V.; Gutman, I.; Zogić, E.; Glogić, E. Resolvent energy of unicyclic, bicyclic and tricyclic graphs. MATCH Commun. Math. Comput. Chem. 2017, 77, 95-104.

35. Liu, H.; Lu, M.; Tian, F. On the spectral radius of unicyclic graphs with fixed diameter. Linear Algebra Appl. 2007, 420, 449-457. [CrossRef] 